\title{
NOTES
}

\section{Identity of V Factor in Culture Medium Used for Prior Growth of Two Strains of Staphylococcus aureus}

\author{
D. F. NIVEN* AND L. LE BLANC $\dagger$ \\ Department of Microbiology, McGill University, Macdonald Campus, 21111 Lakeshore Road, \\ Ste. Anne de Bellevue, Québec, Canada H9X 1 CO
}

\begin{abstract}
Staphylococcus aureus ATCC 12598 and ATCC 25923 were starved of pyridine nucleotides and precursors and then grown in a semidefined medium containing $\left[\right.$ carbonyl $\left.-{ }^{14} \mathrm{C}\right]$ nicotinamide. Samples of medium from late-exponential-phase and stationary-phase cultures were analyzed for ${ }^{14} \mathrm{C}$-metabolites. In all cases, $\mathrm{V}$ factor was present primarily as NAD.
\end{abstract}

$\mathrm{V}$ factor is generally considered to be NAD, although nicotinamide riboside (NR), nicotinamide mononucleotide (NMN), and NADP can also serve as $\mathrm{V}$ factor $(6,9)$. $\mathrm{V}$ factor is required as a nutrient by several, but not all, members of the family Pasteurellaceae, and the satellite phenomenon first described by Grassberger (3) is frequently exploited to determine V-factor dependency $(5,6)$. The test is usually performed with Staphylococcus aureus as the feeder organism $(5,6)$, but other organisms, including pseudomonads $(6,12)$, can also be used (1). Interestingly, however, despite widespread use of this test, to our knowledge the identity of the V-factor compound(s) supplied by these organisms remains unknown.

The purpose of this study was to establish the identity of at least the V-factor compound(s) released by $S$. aureus. This organism is particularly suited for such studies since it requires nicotinic acid (NA) or nicotinamide (NAm) for growth (7), and therefore, radiolabeled NA or NAm can be used to investigate the production of pyridine nucleotides and precursors. Furthermore, Fulton et al. (2) have demonstrated that $S$. aureus fails to assimilate radiolabel from [carbonyl $\left.-{ }^{14} \mathrm{C}\right] \mathrm{NAD},\left[\right.$ carbonyl $\left.-{ }^{14} \mathrm{C}\right] \mathrm{NMN}$, or $\left[\right.$ carbonyl $\left.-{ }^{14} \mathrm{C}\right]$ $\mathrm{NR}$ and hence lacks the corresponding, extracytoplasmic nucleosidases. On the other hand, this organism possesses a membrane-associated pyridine nucleotide pyrophosphatase, and since the organism can assimilate radiolabel from N[U$\left.{ }^{14} \mathrm{C}\right] \mathrm{AD}$, it appears that this pyrophosphatase is oriented extrinsically (2). In effect, while intact organisms are presumably incapable of producing NAm from exogenous $\mathrm{V}$ factor (released by lysed organisms), NAD (and perhaps NADP) may be hydrolyzed by the pyrophosphatase such that $\mathrm{V}$ factor in the culture medium may exist primarily as NMN (or NR) rather than NAD or NADP. Our studies addressed these alternatives.

Two strains of $S$. aureus (strains ATCC 12598 and ATCC 25923) were used. Inocula were prepared and stored as described previously for Haemophilus parasuis (8), except that the growth medium was tryptic soy broth (Difco). To obtain cultures that were starved of pyridine nucleotides and precursors, the organisms were first grown in tryptic soy broth; frozen inocula were thawed rapidly, and $0.2-\mathrm{ml}$ vol-

\footnotetext{
* Corresponding author.

† Present address: Ayerst Laboratories, Montréal, Québec, Canada H4R $1 \mathrm{~J} 6$.
}

umes were used to inoculate duplicate $20-\mathrm{ml}$ volumes of tryptic soy broth contained in $125-\mathrm{ml}$ flasks that were fitted with Morton closures. These cultures were incubated for $4 \mathrm{~h}$ at $37^{\circ} \mathrm{C}$ with agitation on a gyratory shaker $(200 \mathrm{rpm})$. The organisms from these cultures (optical density at $660 \mathrm{~nm}$ [OD 660 ], 2.1; Gilford model 240 spectrophotometer) were harvested by centrifugation at $12,000 \times g$ for $15 \mathrm{~min}$, washed with an equal volume of sterile saline, and resuspended with sterile saline to give an $\mathrm{OD}_{660}$ of approximately 2.5 . Each suspension was then used to initiate a new culture so that two complete sets of data were obtained for each strain. In each case, a $0.2-\mathrm{ml}$ volume of suspension was added to a $125-\mathrm{ml}$ flask (as described above) containing $20 \mathrm{ml}$ of a semidefined medium (SDM); this medium was the medium $51 B$ described by Guirard and Snell (4), except that NA was omitted and the Casamino Acids were vitamin free (Difco). Such cultures in SDM were incubated as described above, except that they were incubated for $11 \mathrm{~h}\left(\mathrm{OD}_{660}, \sim 0.7\right)$, at which time the cultures were well into stationary phase. Each culture was again treated separately. The organisms were harvested and washed as described above and then resuspended with sterile saline to give an $\mathrm{OD}_{660}$ of approx-

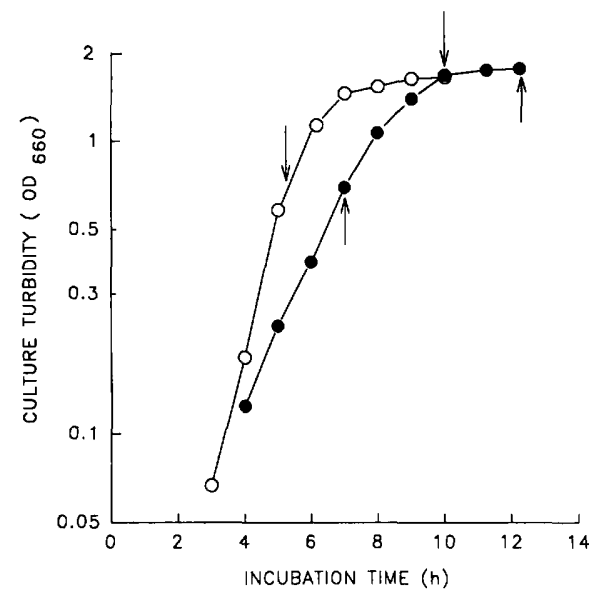

FIG. 1. Growth of $S$. aureus ATCC $12598(\bigcirc)$ and ATCC $25923(\bullet)$ in SDM containing $\left[{ }^{14} \mathrm{C}\right]$ NAm. The arrows $(\downarrow$, strain ATCC 12598; $\uparrow$, strain ATCC 25923) indicate the times at which samples were removed for analysis by high-performance liquid chromatography. 

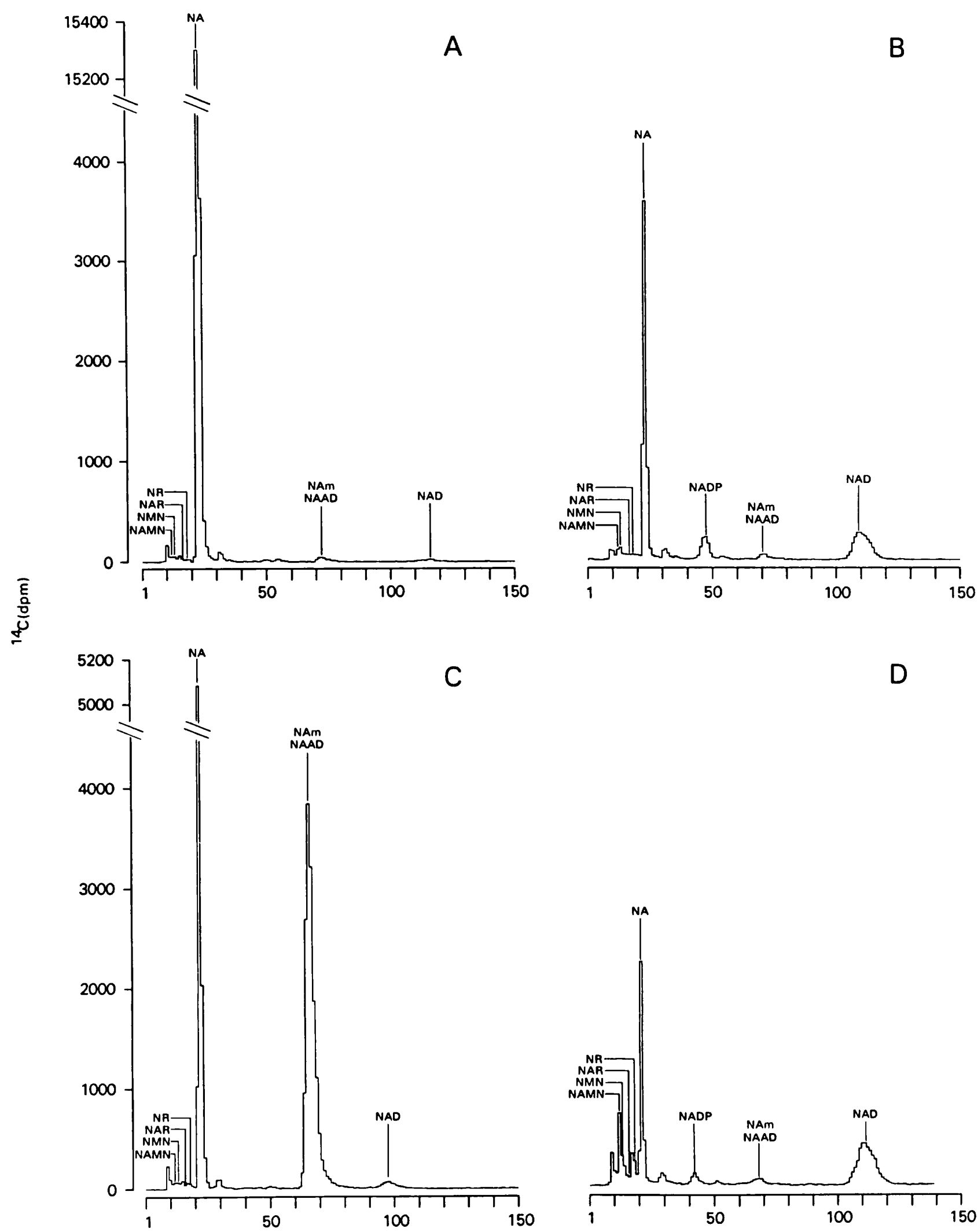

FRACTION NUMBER

FIG. 2. Separation of ${ }^{14} \mathrm{C}$-metabolites in culture medium derived from late-exponential-phase (A and C), and stationary-phase (B and D) cultures of S. aureus ATCC 12598 (A and B) and ATCC 25923 (C and D). The positions (expected or actual) of known compounds are indicated. NAMN, nicotinic acid mononucleotide; NAR, nicotinic acid riboside. 
imately 0.5 , and in each case, a $0.2-\mathrm{ml}$ volume was used to inoculate a $20-\mathrm{ml}$ volume of SDM containing $5 \mu \mathrm{M}$ [carbon$\left.y l-{ }^{14} \mathrm{C}\right] \mathrm{NAm}(40 \mathrm{mCi} / \mathrm{mmol}$; Amersham). The resulting cultures were incubated at $37^{\circ} \mathrm{C}$ with agitation $(200 \mathrm{rpm})$, and growth was monitored turbidimetrically at $660 \mathrm{~nm}$ by using $0.5-\mathrm{ml}$ samples; no growth was observed in comparable experiments in which the NAm was omitted.

Figure 1 shows the growth of the two strains of $S$. aureus in SDM containing $\left[{ }^{14} \mathrm{C}\right] \mathrm{NAm}$; other, comparable sets of data gave essentially identical plots (data not shown). At different times (Fig. 1, arrows), portions $(2 \mathrm{ml})$ of the cultures were removed, and the organisms were sedimented by centrifugation in an Eppendorf centrifuge for $10 \mathrm{~min}$. Each supernatant fraction was then sterilized by filtration and frozen at $-20^{\circ} \mathrm{C}$. The resulting samples almost certainly contained NAD, NMN, or NR since preliminary experiments in which we used $\left[{ }^{12} \mathrm{C}\right] \mathrm{NAm}$ had demonstrated that solid media that were prepared by mixing comparable culture supernatants ( 1 part) and molten 2\% (wt/vol) agar (Difco) in tryptone-yeast extract medium lacking NAD (8) (3 parts) could support the growth of the type strain of Actinobacillus pleuropneumoniae biotype 1 (strain ATCC 27088); this organism, known previously as Haemophilus pleuropneumoniae (11), can use NAD, NMN, or NR as V factor, but it cannot use NADP (10). All samples of culture supernatant were then analyzed by using high-performance liquid chromatography, and the eluates were collected for determinations of radioactivity. The high-performance liquid chromatography system that we used was the system that was described previously (10), in which the mobile phase $\left(0.2 \mathrm{M} \mathrm{NH}_{4} \mathrm{H}_{2} \mathrm{PO}_{4} / \mathrm{NH}_{4} \mathrm{OH}[\mathrm{pH} 6.0]\right.$ containing $0.5 \%$ [vol/ vol] $\left.\mathrm{CH}_{3} \mathrm{OH}\right)$ eluted a $\mu$ Bondapak $\mathrm{C}_{18}$ column $(0.4$ by $15 \mathrm{~cm}$; Waters) at a rate of $2 \mathrm{ml} / \mathrm{min}$. Prior to injection, each sample of culture supernatant $(0.98 \mathrm{ml})$ was mixed with $20 \mu \mathrm{l}$ of a solution containing approximately $2.5 \mathrm{mM}$ NAD, $1 \mathrm{mM}$ $\mathrm{NMN}$, and $1 \mathrm{mM} \mathrm{NR}$; the injection volume was $0.1 \mathrm{ml}$. The eluates were collected in scintillation vials, with each vial containing the eluate collected over a $6-\mathrm{s}$ period. A $5-\mathrm{ml}$ portion of scintillation fluid (Universol; ICN) was added to each vial, and radioactivity was determined by using a Beckman model LS7500 liquid scintillation spectrometer.

Figure 2 shows the elution profiles obtained with the samples referred to in Fig. 1; similar results were obtained with equivalent samples from comparable sets of cultures (data not shown). Interestingly, the cell-free medium derived from cultures of $S$. aureus ATCC 12598 in the late exponential (Fig. 2A) and stationary (Fig. 2B) phases of the growth cycle contained little, if any, NAm, and the predominant ${ }^{14} \mathrm{C}$ metabolite was NA. These results indicate that this organism possesses an extracytoplasmic nicotinamidase and that NA, rather than NAm, may be the transported species. Moreover, it appears that this may be a general feature of $S$. aureus since extracellular fluids derived from late-exponential-phase (Fig. 2C) and stationary-phase (Fig. 2D) cultures of $S$. aureus ATCC 25923 also contained NA. However, the culture fluids from the late-exponential-phase cultures (Fig. 2C) appeared to contain NAm, as well as NA, with NAm predominating; although our high-performance liquid chromatography system could not separate NAm and nicotinic acid adenine dinucleotide (NAAD), the quantities of radiolabeled compound(s) involved, the metabolic implications, and the fact that NAm and/or NAAD was present to only a limited extent in all other samples indicated strongly that the radiolabeled material was indeed NAm rather than NAAD. In effect, it appears that the putative nicotinamidases were produced to different extents by the two strains of $S$. aureus which we studied.
Radiolabeled NAD was detected in all samples, and the concentrations in the culture medium were calculated to be $0.02 \mu \mathrm{M}$ (Fig. 2A), $0.26 \mu \mathrm{M}$ (Fig. 2B), $0.06 \mu \mathrm{M}$ (Fig. 2C), and $0.41 \mu \mathrm{M}$ (Fig. 2D). Interestingly, NADP was present in medium from stationary-phase cultures at concentrations of $0.11 \mu \mathrm{M}$ (Fig. 2B) and $0.06 \mu \mathrm{M}$ (Fig. 2D) but not in medium from late-exponential-phase cultures, and except for medium from stationary-phase cultures of $S$. aureus ATCC 25923 (Fig. 2D), we obtained little, if any, evidence for the presence of NMN or NR. Furthermore, even when NMN and NR appeared to be present, it is possible that the radiolabel was associated with nicotinic acid mononucleotide and nicotinic acid riboside rather than NMN and NR, respectively, because the times at which the acids and amides eluted from the column were separated by only 0.1 and $0.2 \mathrm{~min}$, respectively.

In conclusion, irrespective of the problems outlined above, our data indicate clearly that in culture media derived from liquid cultures of $S$. aureus, $\mathrm{V}$ factor is present primarily as NAD. However, to what extent this is also the case when the organisms are grown on solid medium remains to be established.

This work was supported by the Natural Sciences and Engineering Research Council of Canada.

We thank L. Therrien for providing us with the cultures of $S$. aureus, P. Levert for composing the figures, and M. Parkinson for typing the manuscript.

\section{REFERENCES}

1. Davis, D. J. 1921. The accessory factors in bacterial growth. IV. The "satellite" or symbiosis phenomenon of Pfeiffer's bacillus (B. influenzae). J. Infect. Dis. 29:178-186.

2. Fulton, T., K. Lipski, and M. H. Cynamon. 1988. Inability of Staphylococcus aureus to utilize exogenous NAD accounts for its ability to satellite Haemophilus parainfluenzae, abstr. D-97, p. 87. Abstr. 88th Annu. Meet. Am. Soc. Microbiol. 1988.

3. Grassberger, R. 1897. Beiträge zür Bakteriologie der Influenza. Z. Hyg. Infektionskr. 25:453-475.

4. Guirard, B. M., and E. E. Snell. 1981. Biochemical factors in growth, p. 79-111. In P. Gerhardt, R. G. E. Murray, R. N. Costilow, E. W. Nester, W. A. Wood, N. R. Krieg, and G. B. Phillips (ed.), Manual of methods for general bacteriology. American Society for Microbiology, Washington, D.C.

5. Kilian, M. 1976. A taxonomic study of the genus Haemophilus, with the proposal of a new species. J. Gen. Microbiol. 93:9-61.

6. Kilian, M., and E. L. Biberstein. 1984. Genus II. Haemophilus Winslow, Broadhurst, Krumwiede, Rogers and Smith 1917, p. 558-569. In N. R. Krieg and J. G. Holt (ed.), Bergey's manual of systematic bacteriology, vol. 1. The Williams \& Wilkins Co., Baltimore.

7. Koser, S. A., and F. Saunders. 1938. Accessory growth factors for bacteria and related microorganisms. Bacteriol. Rev. 2:99-160.

8. Niven, D. F. 1984. The cytochrome complement of Haemophilus parasuis. Can. J. Microbiol. 30:763-773.

9. Niven, D. F., and T. O'Reilly. 1990. Significance of V-factor dependency in the taxonomy of Haemophilus species and related organisms. Int. J. Syst. Bacteriol. 40:1-4.

10. O'Reilly, T., and D. F. Niven. 1986. Defining the metabolic and growth responses of porcine haemophili to exogenous pyridine nucleotides and precursors. J. Gen. Microbiol. 132:807-818.

11. Pohl, S., H. U. Bertschinger, W. Frederiksen, and W. Mannheim. 1983. Transfer of Haemophilus pleuropneumoniae and the Pasteurella haemolytica-like organism causing porcine necrotic pleuropneumonia to the genus Actinobacillus (Actinobacillus pleuropneumoniae comb. nov.) on the basis of phenotypic and deoxyribonucleic acid relatedness. Int. J. Syst. Bacteriol. 33:510-514.

12. Shifrine, M., and E. L. Biberstein. 1960. A growth factor for Haemophilus species secreted by a pseudomonad. Nature (London) 187:623. 